\title{
Preparation and characterization of solid lipid nanoparticles loaded with frankincense and myrrh oil
}

Feng Shi

Ji-Hui Zhao

Ying Liu

Zhi Wang

Yong-Tai Zhang

Nian-Ping Feng

School of Pharmacy, Shanghai

University of Traditional

Chinese Medicine, Shanghai,

People's Republic of China
Correspondence: Nian-Ping Feng

Department of Pharmaceutics, School

of Pharmacy, Shanghai University of

Traditional Chinese Medicine,

1200 Cailun Road, Zhangjiang Hi-Tech

Park, Pudong New District, Shanghai

201203, People's Republic of China

Tel +862151322198

Fax +862151322198

Email npfeng@hotmail.com
This article was published in the following Dove Press journal:

International Journal of Nanomedicine

16 April 2012

Number of times this article has been viewed

\begin{abstract}
The aim of the present study was to prepare solid lipid nanoparticles (SLNs) for the oral delivery of frankincense and myrrh essential oils (FMO). Aqueous dispersions of SLNs were successfully prepared by a high-pressure homogenization method using Compritol 888 ATO as the solid lipid and soybean lecithin and Tween 80 as the surfactants. The properties of the SLNs such as particle size, zeta potential (ZP), and drug encapsulation efficiency (EE) were investigated. The morphology of SLNs was observed by transmission electron microscopy (TEM). The crystallinity of the formulation was analyzed by differential scanning calorimetry (DSC) and X-ray diffraction (XRD). In addition, drug evaporation release and antitumor activity were also studied. Round SLNs with a mean size of $113.3 \pm 3.6 \mathrm{~nm}$, a ZP of $-16.8 \pm 0.4 \mathrm{mV}$, and an EE of $80.60 \% \pm 1.11 \%$ were obtained. DSC and XRD measurements revealed that less ordered structures were formed in the inner cores of the SLN particles. Evaporation loss of the active components in FMO could be reduced in the SLNs. Furthermore, the SLN formulation increased the antitumor efficacy of FMO in H22-bearing Kunming mice. Hence, the presented SLNs can be used as drug carriers for hydrophobic oil drugs extracted from traditional Chinese medicines.
\end{abstract}

Keywords: solid lipid nanoparticles, frankincense oil, myrrh oil, evaporation release, antitumor activity, traditional Chinese medicine

\section{Introduction}

Frankincense and myrrh are gum resins obtained from the genera Boswellia and Commiphora, respectively. Both genera belong to the family Burseraceae, which is native to Northeast Africa and the Middle East. ${ }^{1,2}$ Frankincense and myrrh have been used for medical purposes in China and India for thousands of years. ${ }^{3}$ In traditional Chinese medicine, frankincense and myrrh have synergistic effects when used in combination (generally in a 1:1 ratio), and the book Compendium of Materia Medica introduced the therapeutic characteristics of the combination of frankincense and myrrh during the Ming Dynasty. Modern pharmacological research has revealed that essential oils are the primary effective components in frankincense and myrrh oil (FMO) that exhibit a broad spectrum of biological activities such as antimicrobial, anti-inflammatory, and antitumor activities. ${ }^{46}$

However, despite these pharmacological functions, insufficient attention has been focused on the side effects of FMO. As with other essential oils, the instability and poor water solubility of FMO result in poor oral bioavailability, which limits its clinical application. ${ }^{7}$ The components of FMO are sensitive to light, air, and high temperature, and FMO stimulates the gastrointestinal tract, making it unsuitable for oral administration. Therefore, a formulation that can overcome the aforementioned 
problems is highly desired. The conventional method of generating the FMO formulation is beta-cyclodextrin ( $\beta-C D)$ inclusion. Although this method is inexpensive, it has not advanced beyond the empirical stage because the process is cumbersome, and the yielded product is unstable and may contain organic solvent residue. The rapid development of nanodrug delivery systems may support the production of essential oil formulations. Several studies have revealed that nanostructured lipid carriers and self-microemulsifying drug delivery systems enhance the stability and water solubility of essential oils. ${ }^{8,9}$

Solid lipid nanoparticles (SLNs), a new nanoparticlebased drug-delivery system with particles that range in diameter from 10 to $1000 \mathrm{~nm}$, have attracted considerable attention. The advantages of SLNs compared to conventional drug-delivery systems include improved efficacy, reduced toxicity, protection of active compounds, and enhanced biocompatibility..$^{10}$ Moreover, SLNs can be produced on a large scale for oral drug delivery. ${ }^{11}$ Given these features, it was hypothesized that SLNs would be an ideal delivery system for FMO, protecting it from environmental degradation and enhancing its antitumor efficacy. As well as this, a freezedrying procedure following entrapment in SLNs could make FMO more stable and convenient for oral administration.

Therefore, the aim of the present study was to prepare SLNs for the oral delivery of FMO (FMO-SLNs) that are capable of improving the stability and antitumor efficacy of FMO. It must be emphasized that the method for investigating the drug-encapsulation efficiency (EE) of FMO-SLN focuses on specific molecules, and as essential oils such as FMO are complex mixtures of numerous molecules, the use of one or two indexed components is insufficient for comprehensively assessing the EE of FMO-SLNs. Thus, in the present study, the fingerprint similarity (FS) between the total FMO in SLNs and the entrapped FMO in SLNs (stopped by a filter membrane) was determined to evaluate the EE from a macroscopic perspective.

\section{Materials and methods}

\section{Materials}

Compritol 888 ATO $^{\circledR}$ (glyceryl dibehenate/behenate) was donated by Gattefossé (Saint-Priest, France). Tween $80^{\circledR}$ was purchased from Sigma Aldritch (St Louis, MO). Soybean lecithin (Lipoid S 100) was purchased from Lipoid GmbH (Ludwigshafen, Germany). Frankincense and myrrh were purchased from Shanghai Kangqiao Medicine Co, Ltd (Shanghai, China). Octyl acetate (OA; purity $>98 \%$ ) and $\beta$-elemene $(\beta$-E; purity $>98 \%)$ were purchased from the
National Institutes for Food and Drug Control (Beijing, China). 5-Fluorouracil (5-FU) was obtained from Shanghai Xudong Haipu Pharmaceutical Co, Ltd (Shanghai, China). Double-distilled water was obtained through the use of a Millipore ${ }^{\circledR}$ Simplicity System (Millipore, Billerica, MA), and all organic solvents were of analytical reagent grade.

\section{Animal models}

Male Kunming mice of clean grade, weighing 18-22 g, were provided by the Laboratory Animal Center of the Shanghai University of Traditional Chinese Medicine. All experimental procedures were performed in accordance with the approval of the Animal Ethical Committee, Shanghai University of Traditional Chinese Medicine. The animals were kept in an agreeable environment for at least 1 week before the start of the study.

\section{Extraction of FMO}

FMO was extracted using a distillation method. ${ }^{12}$ Eighteen $\mathrm{kg}$ of powdered frankincense and myrrh $(9 \mathrm{~kg}$ each) were distilled in a steam apparatus with an aqueous-phase recycling system for 7 hours. The obtained FMO was dried over sodium sulfate and used as the basic material. FMO was stored at $4^{\circ} \mathrm{C}$ until use.

\section{Gas chromatography spectrometry analysis (GC)}

FMO was analyzed on an Agilent 7890A GC system (Agilent Technologies, Santa Clara, CA) coupled with a flame ionization detector and a split-splitless injector. An Agilent 19091$413 \mathrm{HP}-5$ capillary column $(30 \mathrm{~m} \times 0.32 \mathrm{~mm} \times 0.25 \mu \mathrm{m})$ was used. The temperature of the injector and detector was $250^{\circ} \mathrm{C}$. The speed of the carrier gas (nitrogen) was $2 \mathrm{~mL} / \mathrm{min}$. The temperature was set to increase from $60^{\circ} \mathrm{C}$ to $160^{\circ} \mathrm{C}$ at a rate of $5^{\circ} \mathrm{C} /$ minute and from $160^{\circ} \mathrm{C}$ to $300^{\circ} \mathrm{C}$ at a rate of $10^{\circ} \mathrm{C} /$ minute; the analyte was then isothermally held for 2 minutes at $300^{\circ} \mathrm{C}$. One microliter was injected in the splitless model.

\section{Preparation of FMO-SLNs}

FMO-SLNs were prepared by high-pressure homogenization. ${ }^{13}$ In brief, the lipid phase, consisting of Compritol 888 ATO $(3 \% \mathrm{w} / \mathrm{w})$ and FMO $(2 \% \mathrm{w} / \mathrm{w})$, was heated to $5^{\circ} \mathrm{C}-10^{\circ} \mathrm{C}$ above the melting point of Compritol 888 ATO. The aqueous phase containing a mixture of surfactants (soybean lecithin, $2 \% \mathrm{w} / \mathrm{w}$ and Tween $80,2 \% \mathrm{w} / \mathrm{w}$ ) in double-distilled water was simultaneously prepared at the same temperature. A pre-emulsion was obtained by adding the aqueous phase to the lipid phase through a constant flow pump under stirring 
at $800 \mathrm{rpm}$ for 30 minutes at a temperature above the melting temperature. The hot pre-emulsion was further passed through a high-pressure homogenizer (NS1001L; GEA, Parma, Italy) at 800 bar for six cycles, and the FMO-SLNs were formed after cooling the mixture to room temperature in an ice bath. ${ }^{14}$

\section{Freeze-drying of FMO-SLNs}

Mannitol (10\%) was used as the cryoprotecting agent, and FMO-SLNs in suspension were frozen in a deep freezer (Christ Epsilon 2-4 LSC, Martin Christ Gefriertrocknungsanlagen $\mathrm{GmbH}$, Osterode am Harz, Germany) according to the freeze curve shown in Figure 1. The FMO-SLN powder was then obtained and stored at $4^{\circ} \mathrm{C}$ for DSC and XRD analysis.

\section{Characterization of FMO-SLNs}

\section{Particle size and ZP}

The size and ZP of FMO-SLNs were measured using a Nano ZS90 Zetasizer (Malvern Instruments Ltd, Worcestershire, UK).

\section{$\mathrm{EE}$ and $\mathrm{LC}$}

Free FMO (nonentrapped in the FMO-SLNs) was separated by an ultrafiltration method. ${ }^{15} \mathrm{OA}(21.06 \%$ in FMO $)$ and $\beta$-E ( $2.58 \%$ in FMO) were selected as the indexed components. Centrifugal filter tubes (molecular weight cut-off $=10 \mathrm{kDa}$, Nanosep $^{\circledR}$; Pall Corporation, Port Washington, NY) were used to determine the EE. After a suitable dilution, FMO-SLNs $(0.5 \mathrm{~mL})$ were placed in the upper chamber of a centrifugal filter tube and then centrifuged at $5000 \mathrm{rpm}$ for 15 minutes. The separated part collected at the bottom of the tube was subjected to GC analysis to determine the OA and $\beta$-E content. The total drug content in FMO-SLNs was determined after extraction with dehydrated alcohol in an ultrasonic bath. Drug loading capacity was presented as percent entrapped drug to lipid ratio. Encapsulation efficiency could be calculated by the following equations:

$$
\begin{gathered}
\mathrm{EE}(\%)=\left(\mathrm{EE}_{\mathrm{OA}}+\mathrm{EE}_{\beta-\mathrm{E}}\right) / 2, \\
\mathrm{EE}_{\mathrm{OA}}(\%)=\left(\mathrm{W}_{\text {Total OA }}-\mathrm{W}_{\text {Free OA }}\right) / \mathrm{W}_{\text {Total OA }} \text {, and } \\
\mathrm{EE}_{\beta-\mathrm{E}}(\%)=\left(\mathrm{W}_{\text {Total } \beta-\mathrm{E}}-\mathrm{W}_{\text {Free } \beta-\mathrm{E}}\right) / \mathrm{W}_{\text {Total } \beta-\mathrm{E}}
\end{gathered}
$$

where $\mathrm{W}_{\text {Total OA }}$ and $\mathrm{W}_{\text {Total } \beta \text {-E }}$ are the weights of OA and $\beta$-E in SLNs, respectively, and $\mathrm{W}_{\text {Free OA }}$ and $\mathrm{W}_{\text {Free } \beta \text {-E }}$ are the mean weights of untrapped $\mathrm{OA}$ and $\beta-\mathrm{E}$, respectively.

FMO is composed of hundreds, or even thousands, of components with different molecular structures. The composition of FMO is so complex that the use of only two indexed components did not facilitate comprehensive assessment of the EE of FMO-SLNs. Thus, the FS between the total FMO in SLNs and the entrapped FMO in SLNs (stopped by the filter membrane) was determined to evaluate the EE from a macroscopic point of view. The fingerprint of FMO was obtained using the established GC method mentioned in the section discussing gas chromatography spectrometry analysis, and the method of operation is shown in Figure 2.

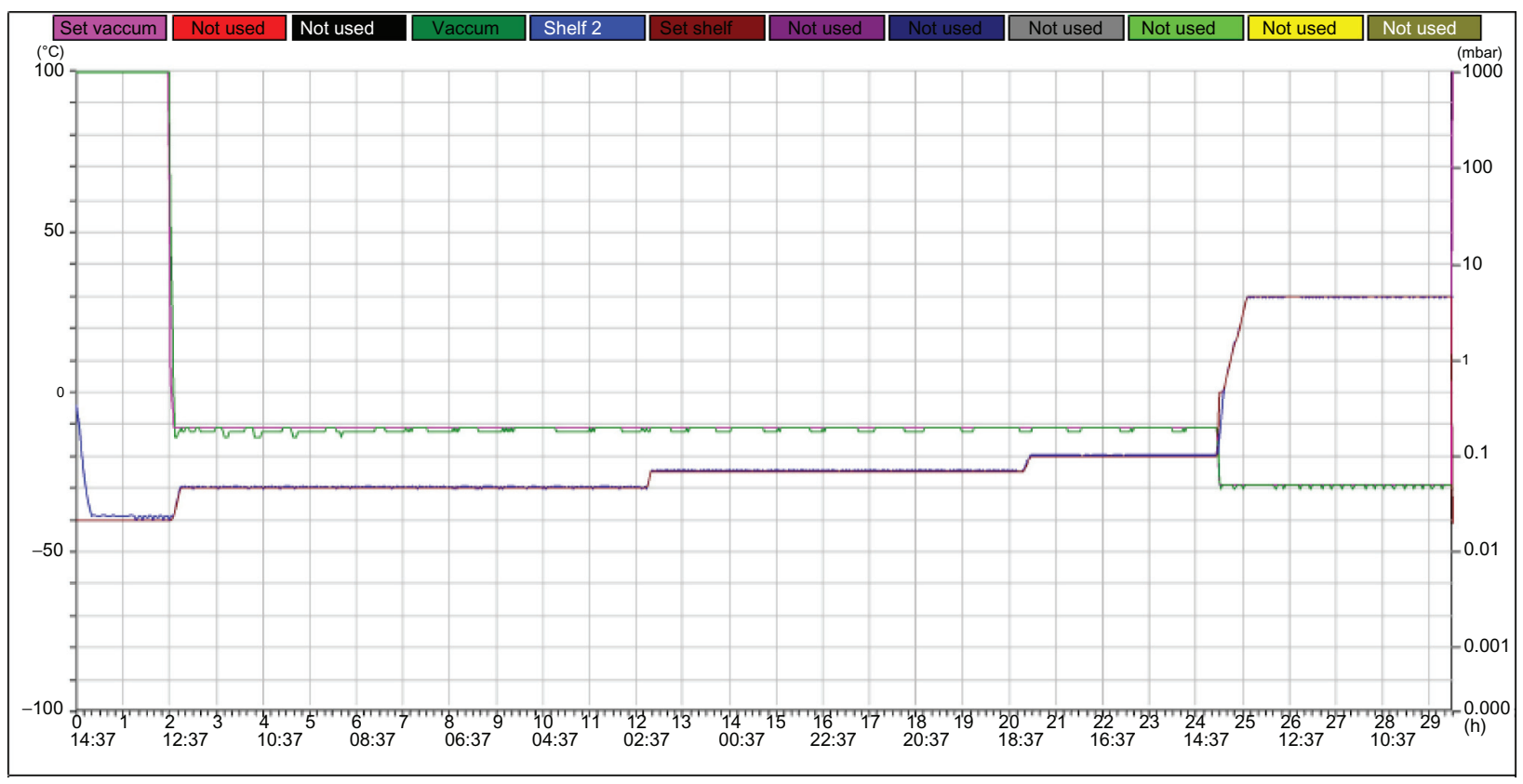

Figure I Freeze-drying curves of FMO-SLNs.

Abbreviation: FMO-SLNs, frankincense and myrrh oil-solid lipid nanoparticles. 


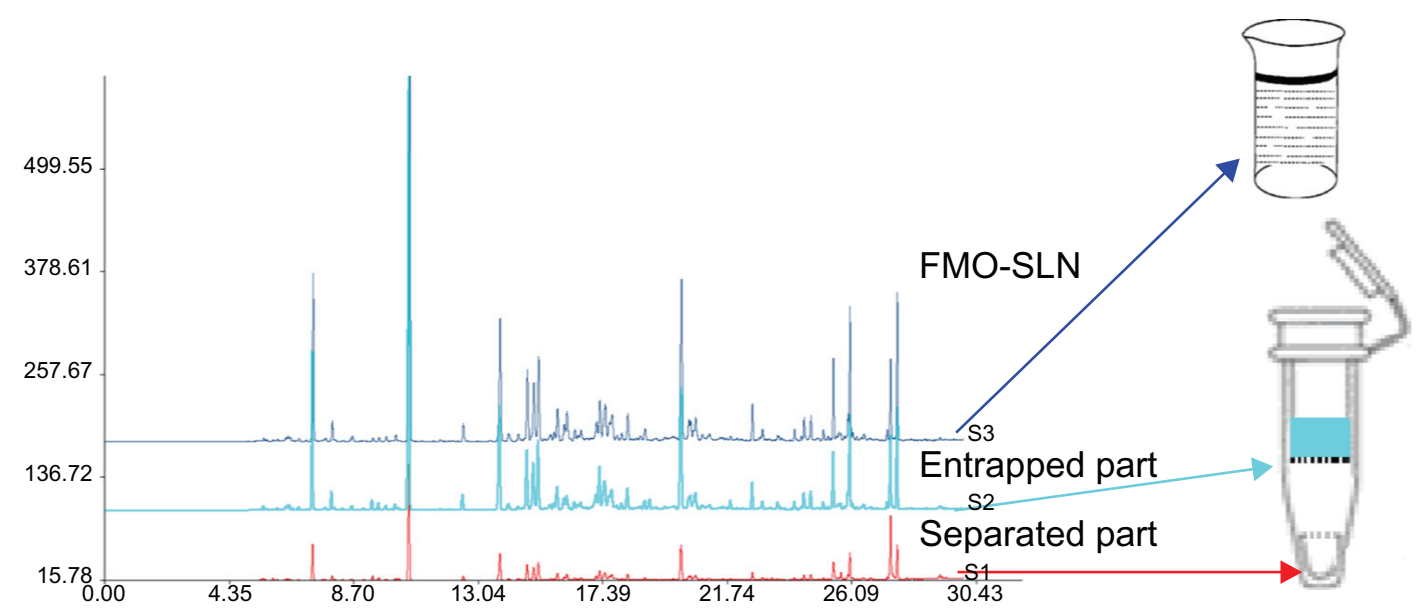

Figure 2 Schematic diagram for evaluation of the EE of FMO-SLNs.

Abbreviations: EE, encapsulation efficiency; FMO-SLNs, frankincense and myrrh oil-solid lipid nanoparticles.

\section{TEM}

The morphology of FMO-SLNs was observed using a transmission scanning microscope (JEM-1230; JEOL, Tokyo, Japan). After a suitable dilution was created, samples were placed on a film-coated copper grid. Thereafter, a drop of $2 \%$ phosphotungstic acid was added to the film and allowed to dry for 10 minutes before observation.

\section{DSC}

DSC was performed using a differential scanning calorimeter (Shimadzu DSC-60; Shimadzu Corporation, Kyoto, Japan). A heating rate of $10^{\circ} \mathrm{C} /$ minute was used, and the temperature range was $20^{\circ} \mathrm{C}-100^{\circ} \mathrm{C}$. An empty aluminum pan was used for reference.

\section{XRD}

Crystalline structures of the FMO-SLN powder were assessed with an X-ray diffractometer (Rigaku Corporation, Tokyo, Japan). A Cu-K $\alpha$ radiation source was used, and samples were scanned over a $2 \theta$ range of $2^{\circ}-50^{\circ}$ with a scanning rate of $5 \%$ minute.

\section{In vitro evaporation release}

To assess the chemical stability of components from unformulated and formulated FMO, samples were placed in open vials and stored at $35^{\circ} \mathrm{C}$ for 6 days. During this time, essential oil was extracted from the samples by immersing them in dehydrated alcohol for 30 minutes in an ultrasonic bath and the selected components were analyzed using the established GC method. Three groups were used: unformulated FMO, FMO- $\beta-C D$, and FMO-SLN. FMO- $\beta-C D$ comprised the $\beta-C D$ inclusion compounds of FMO. FMO- $\beta-C D$ was prepared using a coprecipitation method at a $\beta-\mathrm{CD} / \mathrm{FMO}$ molar ratio of $3: 1{ }^{16}$

\section{In vivo antitumor activity}

H22-bearing Kunming mice were used as a model to assess the in vivo antitumor activity of FMO. ${ }^{17,18} \mathrm{H} 22$ cells were injected and incubated in the abdominal cavity of mice for 6-8 days before they were collected and resuspended in PBS. The collected H22 cells $\left(2 \times 10^{6} / \mathrm{mL}\right)$ were then inoculated subcutaneously into the right forelimb of each mouse at a concentration of $0.2 \mathrm{~mL}$. After injection, the H22-bearing mice were randomly divided into six groups (ten mice/group) that were administered one of the following treatments: saline (control group), 5-FU (reference drug), FMO suspension (prepared with 5\% Tween 80), FMO- $\beta$-CD, blank SLN, or FMO-SLN. 5-FU was administered via intraperitoneal injection once every 2 days at a dose of $25 \mathrm{mg} / \mathrm{kg}$ body weight. All other drugs were administered orally for 10 consecutive days (saline, $0.2 \mathrm{~mL} /$ mouse; FMO suspension, FMO- $\beta$-CD, blank SLN, and FMO-SLN, $100 \mathrm{mg} / \mathrm{kg}$ body weight). After 10 days, the mice were killed, and tumors, spleens, and thymi were excised. The tumor growth inhibition rate was calculated using the following formula: tumor inhibitory rate $(\%)=(\mathrm{C}-\mathrm{T}) / \mathrm{C} \times 100 \%$, where " $\mathrm{C}$ " and " $\mathrm{T}$ " are the average tumor weights in the control and test groups, respectively. The spleen and thymus indices were calculated as follows: spleen/thymus index $=\mathrm{W}_{\mathrm{S} / \mathrm{T}} / \mathrm{W}_{\mathrm{M}}$, where $\mathrm{W}_{\mathrm{S} / \mathrm{T}}$ is the weight of the spleen or thymus and $\mathrm{W}_{\mathrm{M}}$ is the weight of the mouse.

\section{Statistical analysis}

Data are expressed as the mean \pm standard deviation and analyzed using one-way ANOVA. $P$-values less than 0.05 were considered significant. 


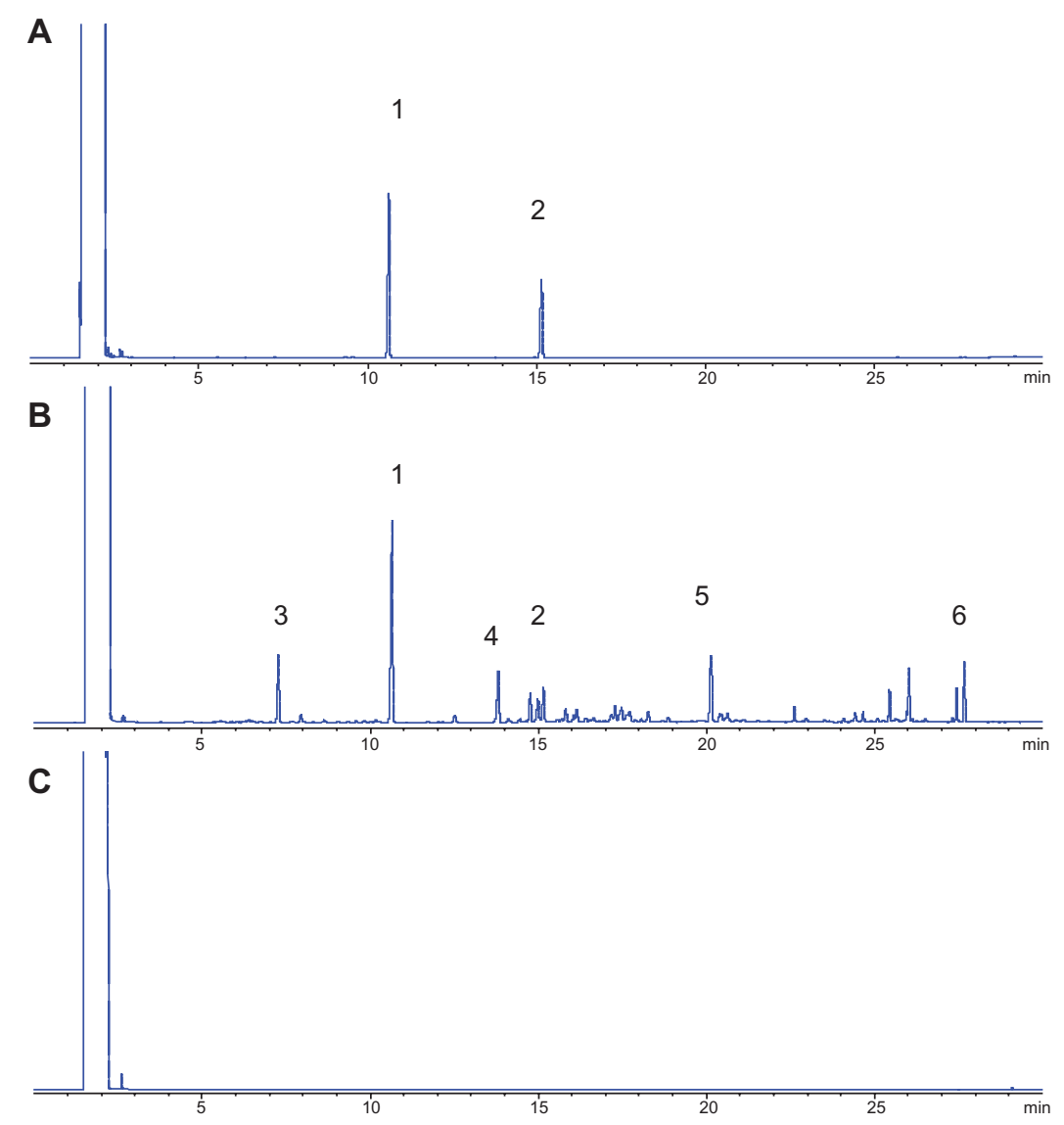

Figure $3 \mathrm{GC}$ of a standard sample (A), FMO-SLN (B), and blank SLN (C); peaks I and 2 correspond to OA and $\beta$-E, respectively. Abbreviations: FMO-SLNs, frankincense and myrrh oil-solid lipid nanoparticles; GC, gas chromatography; OA, octyl acetate; $\beta$-E, $\beta$-elemene.

\section{Results and discussion Extraction and GC analysis}

A green essential oil (FMO) was obtained at a good yield of $0.83 \%$ by distillation. The established gas chromatograms are shown in Figure 3, which indicates that the selected indexed components (from peaks 1 to 6 ) could be separated under the GC condition, and that the pharmaceutical adjuvants (including Compritol 888 ATO, Tween 80, and soybean lecithin) did not influence the analysis. The gas chromatograms of FMO revealed that FMO is a complex mixture of organic compounds. Among the marked peaks, peaks 1 and 2 correspond to $\mathrm{OA}$ and $\beta$-E, respectively. OA is present at the highest concentration $(21.06 \%)$ in FMO, and $\beta$-E $(2.58 \%)$ exerts a favorable antitumor activity; ${ }^{19-21}$ therefore, these compounds were chosen as the indexed components. Peaks 1 to 6 were used to assess the in vitro evaporation release of unformulated and formulated FMO.

\section{Preparation of FMO-SLNs}

Production techniques for SLNs include high-pressure homogenization, ${ }^{22}$ microemulsion, ${ }^{23}$ an ultrasound-based method, ${ }^{24}$ and solvent emulsification/evaporation. ${ }^{25,26}$ In the present study, SLNs were prepared by high-pressure homogenization. High-pressure homogenization is a reliable and powerful technique for the preparation of SLNs, and it has been utilized on a large scale in the pharmaceutical industry. In our preliminary study, FMO was found to have high solubility in different lipids, such as Compritol 888 ATO, Precirol ATO 5, glycerin monostearate, and stearin. However, a high EE was obtained using Compritol 888 ATO as the lipid. A mixture of soybean lecithin and Tween 80 was used as the surfactant. Other details of the SLN formulations are given in Table 1.

\section{Characterization of FMO-SLNs}

Figure 4A shows the typical particle size distribution of FMO-SLNs. As revealed in this figure, FMO-SLNs were

Table I Sample composition (\%) of FMO-SLNs (w/w)

\begin{tabular}{llllll}
\hline Formulation & $\begin{array}{l}\text { Compritol } \\
\text { 888 ATO }\end{array}$ & FMO & $\begin{array}{l}\text { Soybean } \\
\text { lecithin }\end{array}$ & Tween $\mathbf{8 0}$ & Water \\
\hline SLN & 3 & 2 & 2.5 & 2.5 & 90 \\
\hline
\end{tabular}

Abbreviation: FMO-SLNs, frankincense and myrrh oil-solid lipid nanoparticles. 

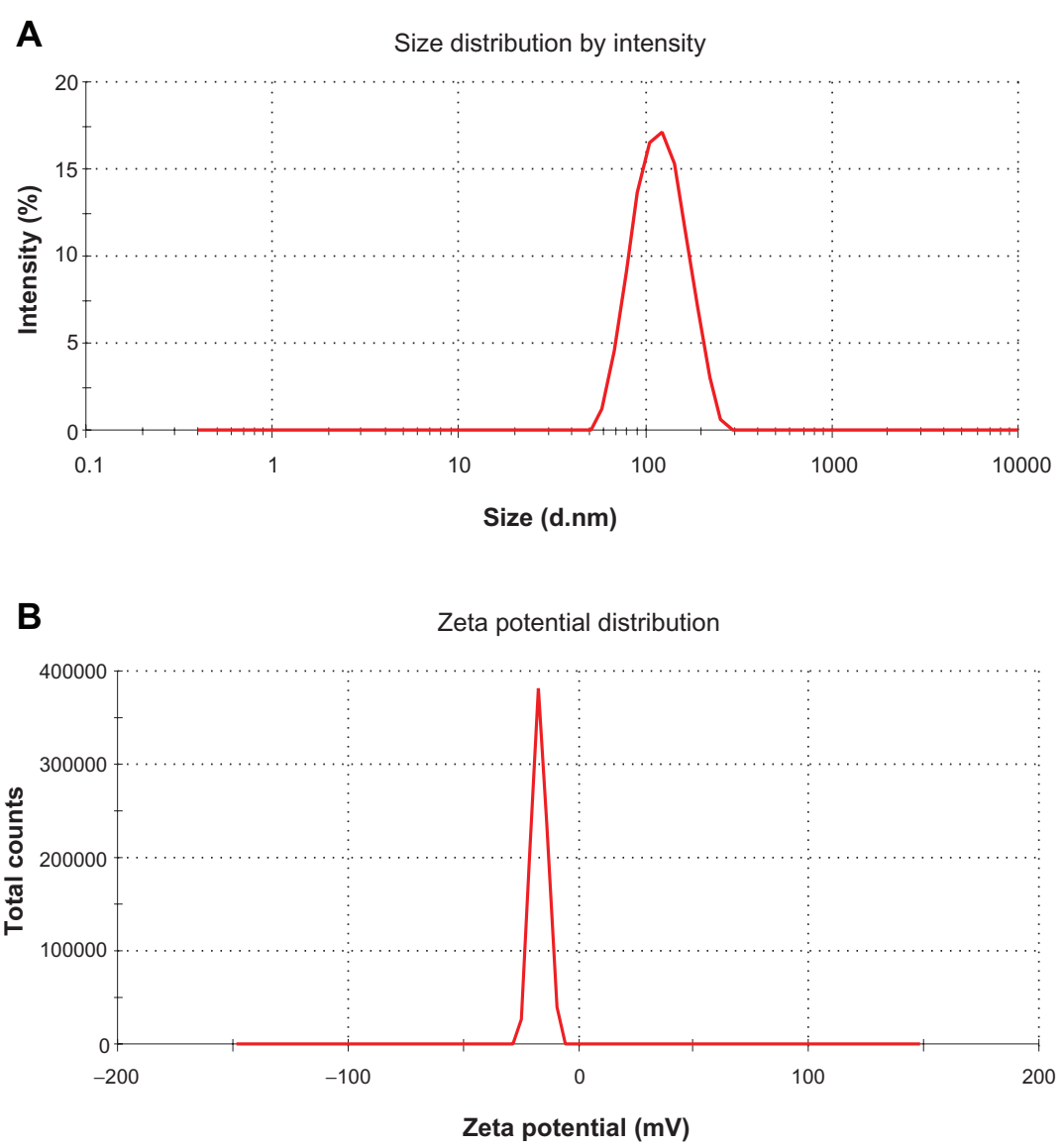

Figure 4 Particle size (A) and ZP (B) of FMO-SLNs.

Abbreviations: FMO-SLNs, frankincense and myrrh oil-solid lipid nanoparticles; ZP, zeta potential.

Table 2 EE and LC of SLNs $(n=3)$

\begin{tabular}{llllll}
\hline SLN & EE $_{\mathrm{OA}}(\%)$ & $\mathrm{EE}_{\beta-\mathrm{E}}(\%)$ & $\mathrm{EE} \mathrm{( \% )}$ & LC (\%) & FS (\%) \\
\hline NO I & 81.8 & 80.2 & 81.00 & 54.0 & 98.93 \\
NO 2 & 83.6 & 75.1 & 79.35 & 52.9 & 98.03 \\
NO 3 & 84.3 & 78.6 & 81.45 & 54.3 & 99.09 \\
Mean & $83.23 \pm 1.29$ & $77.97 \pm 2.61$ & $80.60 \pm 1.11$ & $53.73 \pm 0.74$ & $98.68 \pm 0.57$ \\
\hline
\end{tabular}

Abbreviations: $E E$, encapsulation efficiency; FS, fingerprint similarity; LC, loading capacity; OA, octyl acetate; SLNs, solid lipid nanoparticles; $\beta$-E, $\beta$-elemene.
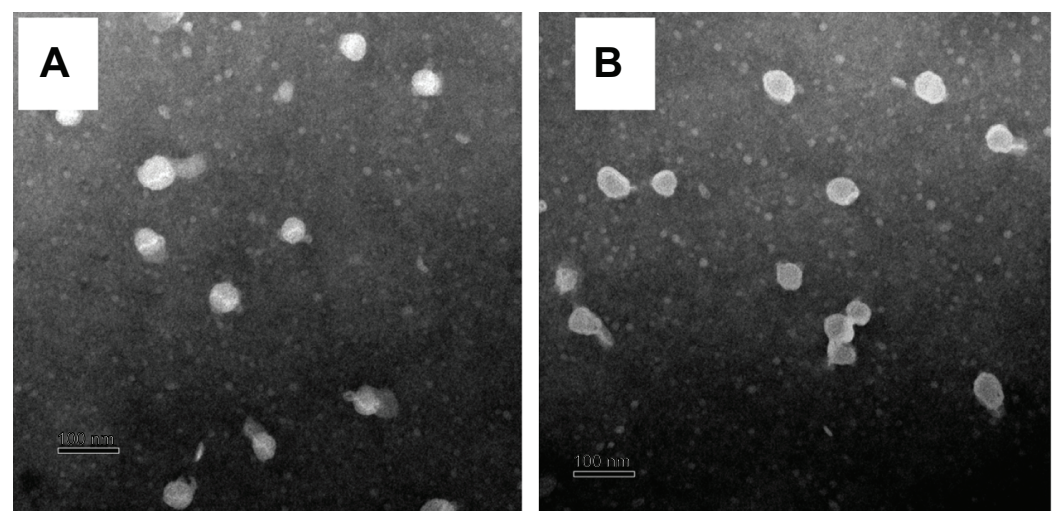

Figure 5 TEM images of the blank SLNs (A) and FMO-SLNs (B).

Abbreviations: FMO-SLNs, frankincense and myrrh oil-solid lipid nanoparticles; TEM, transmission electron microscopy. 


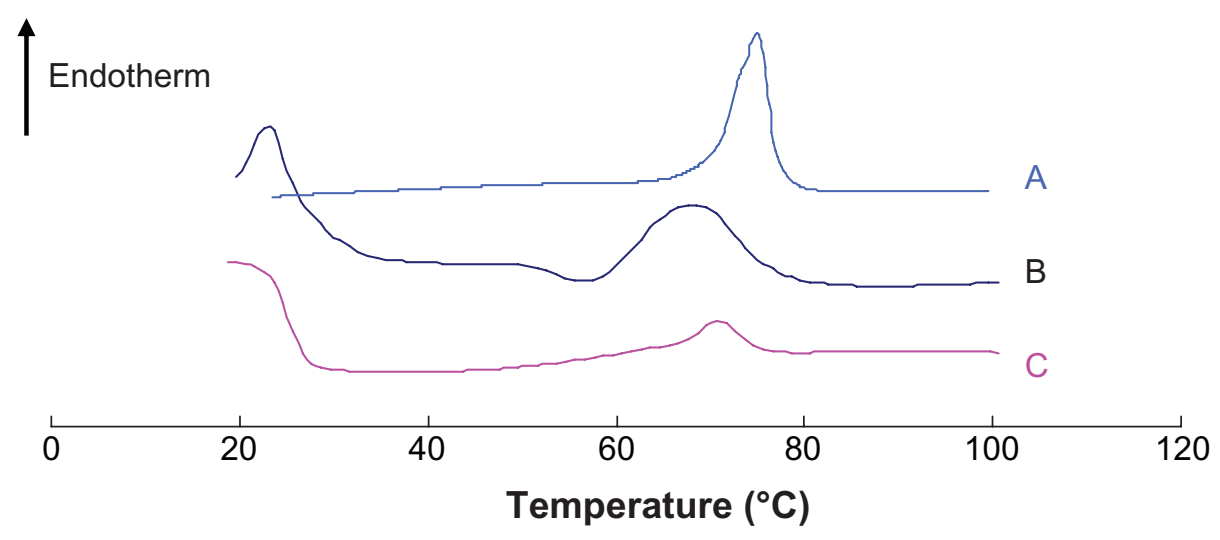

Figure 6 DSC curves of (A) Compritol 888 ATO, (B) physical mixture, and (C) FMO-SLNs. Abbreviations: DSC, differential scanning calorimetry; FMO-SLNs, frankincense and myrrh oil-solid lipid nanoparticles.

found to be nanometric and unimodal with a relatively narrow size distribution that ranged from 43.8 to $219.5 \mathrm{~nm}$. The average diameter was $113.3 \pm 3.6 \mathrm{~nm}$, and the polydispersity index value was 0.25 . ZP is also an important surface characteristic of SLNs, and the measurement of ZP allows predictions of the storage stability of SLNs. ${ }^{27}$ As revealed in Figure 4B, the FMO-SLNs possessed a high ZP with the value of $-16.8 \pm 0.4 \mathrm{mV}$, indicating that the surface of the FMO-SLNs was negatively charged.

$\mathrm{EE}$ is a very important characteristic for judging the quality of SLNs. Generally, the methods of assessing EE include ultracentrifugation, ${ }^{28}$ Sephadex minicolumn centrifugation, ${ }^{29}$ dialysis, ${ }^{30}$ and the ultrafiltration method used in the present study. As mentioned in the section on extraction and GC analysis, FMO is a complex mixture of abundant compounds. Therefore, two components were selected as indexed components to evaluate the EE from a microscopic point of view, and the FS between the total FMO in SLNs and the entrapped FMO in SLNs was determined to evaluate the EE from a macroscopic point of view. As is shown in Table 2, a high incorporation capacity of SLNs was observed because of the high lipophilicity of FMO and its good compatibility with Compritol 888 ATO. The FS was $98.68 \% \pm 0.57 \%$, indicating that the most abundant components were entrapped by the SLNs. The results of the present study confirm those of previous work, indicating that hydrophobic drugs could be incorporated well into lipid carriers with high EE. ${ }^{31}$

The morphologies of the blank SLNs and FMO-SLNs were observed by TEM. As shown in Figure 5, most of the particles were round and uniform in size. No significant difference was found between the blank SLNs and FMO-SLNs.
As has been reported previously, ${ }^{32,33}$ DSC can be used to determine thermodynamic variations related to morphological changes, because different lipid modifications possess different melting points and melting enthalpies. In the present study, DSC was performed to investigate the melting and crystallization behavior of the lipid matrices to detect whether these characteristics were changed by SLN preparation. As shown in Figure 6, the bulk lipid (Compritol 888 ATO) displayed a melting point of $75^{\circ} \mathrm{C}$. The physical mixture and SLN heating curves differed distinctly from those of the bulk lipid, and shifts of the melting point of the physical mixture and SLNs to $68.3^{\circ} \mathrm{C}$ and $70.9^{\circ} \mathrm{C}$, respectively, were observed. The reduction of the melting point of the physical mixture was likely caused by the lowboiling-point components in FMO. Table 3 presents the melting points, enthalpy, and crystallinity of the samples. The enthalpy and crystallinity of FMO-SLNs were lower than those of the initial lipid, indicating an increased number of lattice defects in FMO-SLNs. ${ }^{34}$ The results indicate that the perfect crystal structure of Compritol 888 ATO was changed by SLN preparation, and a new less-ordered phase was formed.

As a further microstructure investigation of FMOSLNs, XRD was performed to confirm the findings obtained by DSC. The X-ray patterns of Compritol

Table 3 Melting points, enthalpy, and crystallinity of each sample

\begin{tabular}{llll}
\hline Samples & $\begin{array}{l}\text { Melting points } \\
\left({ }^{\circ} \mathbf{C}\right)\end{array}$ & $\begin{array}{l}\text { Enthalpy } \\
(\mathbf{J} / \mathbf{g})\end{array}$ & $\begin{array}{l}\text { Crystallinity } \\
(\%)\end{array}$ \\
\hline Lipid & 75.0 & 130.7 & 100.0 \\
Physical mixture & 68.3 & 138.4 & 105.9 \\
FMO-SLNs & 70.9 & 27.2 & 20.8
\end{tabular}

Abbreviation: FMO-SLNs, frankincense and myrrh oil-solid lipid nanoparticles. 


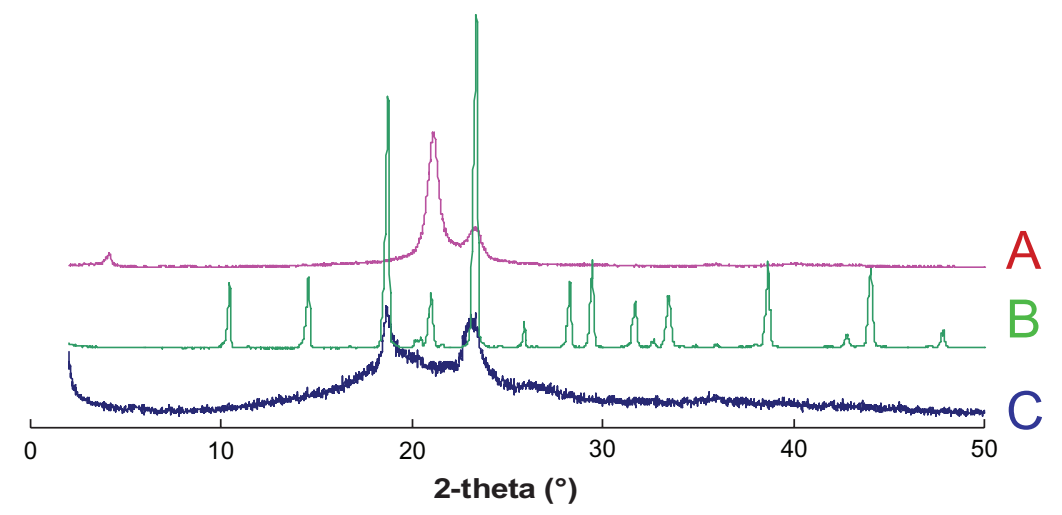

Figure 7 X-ray spectra of (A) Compritol 888 ATO, (B) mannitol, and (C) FMO-SLNs. Abbreviation: FMO-SLNs, frankincense and myrrh oil-solid lipid nanoparticles.

888 ATO, mannitol, and FMO-SLNs are shown in Figure 7. As indicated in Figure 7A, the bulk Compritol 888 ATO displayed three peaks at positions $2 \theta=4.16^{\circ}, 21.16^{\circ}$, and $23.30^{\circ}$, while Figure $7 \mathrm{C}$ shows that the peaks at $2 \theta=4.16^{\circ}$ and $21.16^{\circ}$ disappeared. In Figure $7 \mathrm{C}$ two blunt peaks appeared that were likely influenced by the cryoprotectant (mannitol), which has a high degree of crystallinity (Figure 7B). From these results, it was confirmed that Compritol 888 ATO exhibited a less-ordered structure in SLNs.

\section{In vitro evaporation release}

Generally, essential oils are unstable when exposed to air, light, and high temperature, which may result in the rapid evaporation and degradation of some active components and further lead to a dramatic decrease in activity. ${ }^{35}$ Several studies have demonstrated that the incorporation of essential oils into SLNs helps to increase their stability. ${ }^{36,37}$ To investigate the capability of SLNs to prevent the rapid evaporation of FMO, six components that possess different boiling points according to the established GC spectrum were chosen as the indexed components. $\beta-C D$ inclusion is the most common technique to incorporate essential oils, and thus, in the present study, the evaporation release behavior of FMO- $\beta$-CD was determined as a control. The data for the in vitro evaporation release of unformulated FMO, FMO- $\beta-C D$, and FMO-SLNs are presented in Table 4. Table 4A clearly shows the rapid evaporation loss of all six selected components from the initial FMO, with evaporation beginning on the second day with large losses of more than 50\% seen after 6 days of storage (excluding component 4). Compared with the initial FMO, FMO- $\beta$-CD (Table 4B) and FMO-SLN (Table 4C) exhibited a considerable decrease in evaporation loss, and no component exhibited an evaporation loss exceeding 50\% after 6 days of storage, indicating that both $\beta$-CD inclusion and SLN incorporation can prevent the evaporation loss of components in FMO.

\section{In vivo antitumor activity}

H22 tumor-bearing mice were used as an animal model to assess whether FMO-SLNs exhibit antitumor activity. As shown in Table 5, neither saline nor blank SLN had any measurable effect on tumor growth, indicating that the SLN

Table 4 Evaporation release of components

\begin{tabular}{|c|c|c|c|c|c|c|}
\hline Percent loss (\%) & No I & No 2 & No 3 & No 4 & No 5 & No 6 \\
\hline \multicolumn{7}{|c|}{ A. Initial FMO $(n=3)$} \\
\hline Day I & 0 & 0 & 0 & 0 & 0 & 0 \\
\hline Day 2 & 12.22 & $|2.5|$ & 13.39 & 5.15 & 28.24 & 48.96 \\
\hline Day 3 & 19.99 & 18.58 & 18.13 & 18.48 & 33.03 & 55.05 \\
\hline Day 4 & 26.28 & 25.31 & 25.65 & 23.13 & 39.05 & 57.78 \\
\hline Day 5 & 38.56 & 37.28 & 36.82 & 34.17 & 48.5 & 63.98 \\
\hline Day 6 & 50.92 & 50.07 & 50.01 & 46.1 & 58.08 & 71.09 \\
\hline \multicolumn{7}{|c|}{ B. FMO- $\beta-C D(n=3)$} \\
\hline Day I & 0 & 0 & 0 & 0 & 0 & 0 \\
\hline Day 2 & -3.13 & -1.64 & 0.43 & -4.9 & -0.07 & 2.91 \\
\hline Day 3 & 0.9 & 4.27 & 10.01 & -0.29 & 6.54 & 2.58 \\
\hline Day 4 & 13.98 & 20.05 & 29.71 & 28.27 & 36.05 & 29.47 \\
\hline Day 5 & 17.44 & 21.37 & 31.63 & 26.44 & 41.13 & 31.65 \\
\hline Day 6 & 18.74 & 22.95 & 33.87 & 27.69 & 42.59 & 34.67 \\
\hline \multicolumn{7}{|c|}{ C. FMO-SLNs $(n=3)$} \\
\hline Day I & 0 & 0 & 0 & 0 & 0 & 0 \\
\hline Day 2 & 8.21 & 6.69 & 4.54 & 7.16 & 7.31 & 4.34 \\
\hline Day 3 & 19.9 & 13.53 & 11.15 & 15.6 & 15.13 & 8.55 \\
\hline Day 4 & $28.4 I$ & 20.73 & 17.39 & 18.68 & 19.57 & 11.59 \\
\hline Day 5 & 37.57 & 24.6 & 21.46 & 23.87 & 21.16 & 13.88 \\
\hline Day 6 & 44.47 & 30.93 & 22.02 & 26.14 & 26.16 & 14.73 \\
\hline
\end{tabular}

Abbreviation: FMO-SLNs, frankincense and myrrh oil-solid lipid nanoparticles. 
Table 5 In vivo antitumor effects in H22-bearing mice $(n=10)$

\begin{tabular}{lllll}
\hline Drug groups & \multicolumn{2}{l}{ Mice body weight $(\mathrm{g})$} & Tumor weight $(\mathrm{g})$ & Inhibition rate $(\%)$ \\
\cline { 2 - 4 } & Before study & After study & & \\
\hline Saline & $19.84 \pm 1.21$ & $30.39 \pm 3.24$ & $1.88 \pm 0.21$ & - \\
$5-\mathrm{FU}(25 \mathrm{mg} / \mathrm{kg})$ & $19.17 \pm 1.13$ & $24.47 \pm 2.85$ & $0.59 \pm 0.17 * *$ & 68.55 \\
Blank SLN & $19.42 \pm 1.36$ & $30.94 \pm 4.03$ & $1.81 \pm 0.22$ & 3.68 \\
FMO $(100 \mathrm{mg} / \mathrm{kg})$ & $20.03 \pm 1.55$ & $25.13 \pm 2.31$ & $1.26 \pm 0.24 * *$ & 32.94 \\
$\beta-C D(100 \mathrm{mg} / \mathrm{kg})$ & $19.38 \pm 0.99$ & $26.11 \pm 2.50$ & $1.22 \pm 0.16 * *$ & 34.8 \\
SLN $(100 \mathrm{mg} / \mathrm{kg})$ & $19.29 \pm 1.37$ & $25.23 \pm 3.18$ & $1.06 \pm 0.15 * * . \#$ & 43.66 \\
\hline
\end{tabular}

Notes: FMO-SLNs, frankincense and myrrh oil-solid lipid nanoparticles. $* * P<0.01$ vs saline control group; ${ }^{*} P<0.05$ vs $\mathrm{FMO}$ suspension group, $\beta$-CD group, and FMO- $\beta$-CD group at $100 \mathrm{mg} / \mathrm{kg}$ body weight.

Abbreviation: FMO-SLNs, frankincense and myrrh oil-solid lipid nanoparticles.

vehicle had no influence on antitumor activity. Compared with the findings for the saline group, significant antitumor effects $(P<0.01)$ were observed for the FMO suspension, FMO- $\beta-C D$, and FMO-SLNs. The tumors in these three groups were considerably smaller than those in the saline group. The inhibition rates of the FMO suspension, FMO$\beta-\mathrm{CD}$, and FMO-SLNs (at a concentration of $100 \mathrm{mg} / \mathrm{kg}$ ) were $31.23 \%, 34.81 \%$, and $43.66 \%$, respectively. These results indicate that the inhibition rate of FMO- $\beta-C D$ against tumor growth was similar to that of the FMO suspension $(P>0.05)$, and that the slight enhancement of the inhibition rate may be attributable to the increased dissolution of FMO by $\beta-C D$ inclusion. These findings also suggest that the antitumor efficacy of the FMO-SLNs was significantly higher $(P<0.05)$ than that of the FMO suspension and FMO- $\beta$-CD at the same dosage. These results confirmed that SLNs represent a better delivery system for antitumor therapy.

Changes in the body weight of H22-bearing mice over the course of the study are also presented in Table 5. The weight of the mice in the saline and blank SLN groups increased rapidly, which may have been caused by tumor growth. The rates of the weight increase in the 5-FU and FMO-SLN groups were lower than those in the saline group.

The spleen and thymus are the two primary immune organs related to antitumor activity. ${ }^{38}$ As shown in Figure 8, the 5-FU group exhibited significant reductions $(P<0.05)$ in both the spleen and thymus indices as compared to the saline group. Slight enhancements of these indices were observed in the FMO suspension, FMO- $\beta-C D$, and FMO-SLN groups, indicating that FMO could enhance the immune response in tumor-bearing mice. The results confirmed the previous findings that traditional Chinese medicines can exert antitumor effects on the immune system. ${ }^{39}$

Numerous investigations have shown that SLNs can increase the antitumor efficacy of drugs while reducing their systemic side effects. ${ }^{40}$ This may be due to the controlled release behavior and targetable distribution of SLNs. A future study will focus on the organ distribution of FMO-SLNs.

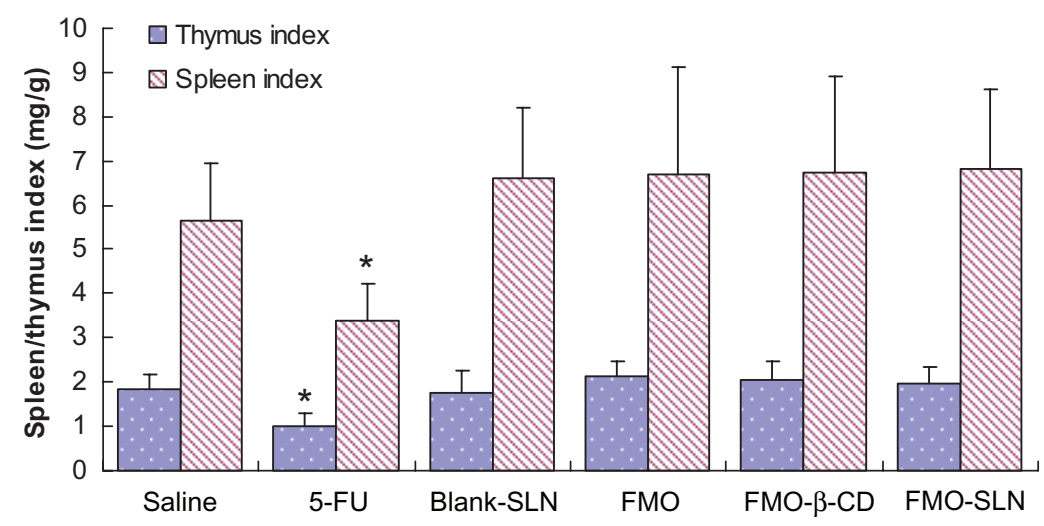

Figure 8 Evaluation of the thymus and spleen indices in tumor-bearing mice.

Note: ${ }^{*} P<0.05$ compared with the saline group. 


\section{Conclusion}

In this study, FMO-SLNs were successfully prepared by high-pressure homogenization. Compritol 888 ATO showed reasonable FMO solubilization capacity. The poorly watersoluble drug FMO was efficiently encapsulated into the nanoparticles. Particles prepared under proper formulation conditions were spherical with diameters of $<220 \mathrm{~nm}$. A high EE was obtained using the described method. Physicochemical characterization revealed that efficiently prepared SLNs encapsulated the drug. The drug evaporation release study showed that SLN incorporation could prevent the evaporation loss of FMO components to a desirable degree. Furthermore, FMO-SLNs possess significantly higher antitumor efficacy. Hence, the developed SLNs can be used to increase the stability and/or to improve the in vivo antitumor efficacy of FMO. However, further studies should focus on the absorption and distribution of the FMO-SLNs.

\section{Acknowledgments}

This work was financially supported by the Subject Chief Scientist Program (10XD14303900) from the Science and Technology Commission of Shanghai Municipality, and the Program (NCET08-0898) for New Century Excellent Talents and Program (No IRT1071) for Changjiang Scholars and Innovative Research Team in Universities from the State Education Ministry, PR China.

The authors gratefully acknowledge Dr Zhenghai Zhang of Jiangsu Provincial Academy of Chinese Medicine for his helpful suggestions.

\section{Disclosure}

The authors report no conflicts of interest in this work.

\section{References}

1. Frank A, Unger M. Analysis of frankincense from various Boswellia species with inhibitory activity on human drug metabolising cytochrome P450 enzymes using liquid chromatography mass spectrometry after automated on-line extraction. J Chromatogr A . 2006;1112(1-2):255-262.

2. Massoud A, El-Sisi S, Salama O, Massoud A. Preliminary study of therapeutic efficacy of a new fasciolocidal drug derived from Commiphora molmol (myrrh). Am J Trop Med Hyg. 2001;65(2):96-99.

3. Döhling C. Boswellia serrata (Frankincense)-from traditional Indian medicine (Ayurveda) to evidence-based medicine. Phytomedicine. 2008; 15(6):540.

4. Van Vuuren SF, Kamatou GPP, Viljoen AM. Volatile composition and antimicrobial activity of twenty commercial frankincense essential oil samples. S Afr J Bot. 2010;76(4):686-691.

5. Tipton DA, Lyle B, Babich H, Dabbous MKh. In vitro cytotoxic and anti-inflammatory effects of myrrh oil on human gingival fibroblasts and epithelial cells. Toxicol in Vitro. 2003;17(3):301-310.

6. Ashry KM, El-Sayed YS, Khamiss RM, El-Ashmawy IM. Oxidative stress and immunotoxic effects of lead and their amelioration with myrrh (Commiphora molmol) emulsion. Food Chem Toxicol. 2010;48(1): 236-241.
7. Pillmoor JB, Wright K, Terry AS. Natural products as a source of agrochemicals and leads for chemical synthesis. Pestic Sci. 1993;39(2): 131-140.

8. Zhao XL, Yang CR, Yang KL, Li KX, Hu HY, Chen DW. Preparation and characterization of nanostructured lipid carriers loaded traditional Chinese medicine, zedoary turmeric oil. Drug Dev Ind Pharm. 2010; 36(7):773-780.

9. Yao G, Li Y. Preparation, characterization and evaluation of selfmicroemulsifying drug delivery systems (SMEDDSs) of Ligusticum chuanxiong oil. Biomed Pharmacother. 2011;1(1):36-42.

10. Souto EB, Müller RH. Lipid nanoparticles: effect on bioavailability and pharmacokinetic changes. Handb Exp Pharmacol. 2010;197: 115-141.

11. Müller RH, Mäder K, Gohla S. Solid lipid nanoparticles (SLN) for controlled drug delivery - a review of the state of the art. Eur J Pharm Biopharm. 2000;50(1):161-177.

12. Sharma M, Agrawal SK, Sharma PR, et al. Cytotoxic and apoptotic activity of essential oil from Ocimum viride towards COLO 205 cells. Food Chem Toxicol. 2010;48(1):336-344.

13. Silva AC, González-Mira E, García ML, et al. Preparation, characterization and biocompatibility studies on risperidone-loaded solid lipid nanoparticles (SLN): high pressure homogenization versus ultrasound. Colloids Surf B Biointerfaces. 2011;86(1):158-165.

14. Ridolfi DM, Marcato PD, Justo GZ, Cordi L, Machado D, Durán N. Chitosan-solid lipid nanoparticles as carriers for topical delivery of tretinoin. Colloids Surf B Biointerfaces. 2012;93:36-40.

15. Liu Y, Wang PF, Sun C, et al. Wheat germ agglutinin-grafted lipid nanoparticles: Preparation and in vitro evaluation of the association with Caco-2 monolayers. Int J Pharm. 2010;397(1-2):155-163.

16. Li XH, Jin ZY, Wang J. Complexation of allyl isothiocyanate by $\alpha$ - and $\beta$-cyclodextrin and its controlled release characteristics. Food Chem. 2007;103(2):461-466.

17. Zhang J, Wang X, Lu H. Amifostine increases cure rate of cisplatin on ascites hepatoma 22 via selectively protecting renal thioredoxin reductase. Cancer Lett. 2008;260(1-2):127-136.

18. Lou H, Gao L, Wei X, et al. Oridonin nanosuspension enhances antitumor efficacy in SMMC-7721 cells and H22 tumor bearing mice. Colloids Surf B Biointerfaces. 2011;87(2):319-325.

19. Yao YQ, Ding X, Jia YC, Huang CX, Wang YZ, Xu YH. Anti-tumor effect of beta-elemene in glioblastoma cells depends on p38 MAPK activation. Cancer Lett. 2008;264(1):127-134.

20. Sun YH, Liu GF, Zhang YQ, Zhu H, Ren Y, Shen YM. Synthesis and in vitro anti-proliferative activity of $\beta$-elemene monosubstituted derivatives in HeLa cells mediated through arrest of cell cycle at the G1 phase. Bioorg Med Chem. 2009;17(3):1118-1124.

21. Xu LY, Tao SJ, Wang XM, et al. The synthesis and anti-proliferative effects of beta-elemene derivatives with mTOR inhibition activity. Bioorg Med Chem. 2006;14(15):5351-5356.

22. Müller RH, Mehnert W, Lucks JS, et al. Solid lipid nanoparticles (SLN) an alternative colloidal carrier system for controlled drug delivery. Eur J Pharm Biopharm. 1995;41(1):62-69.

23. Gasco MR. Solid lipid nanospheres from warm micro-emulsions. Pharm Technol Eur. 1997;9(11):52-58.

24. Speiser P. Lipidnanopellets als tragersystem fur Arzneimittel zur peroralen anwendung. European Patent EP 0167825. November 14, 1989.

25. Sjöstörm B, Bergenståhl B. Preparation of submicron drug particles in lecithin-stabilized o/w emulsions. I. Model studies of the precipitation of cholesteryl acetate. Int J Pharm. 1992;84(2):107-116.

26. Siekmann B, Westesen K. Investigations on solid lipid nanoparticles prepared by precipitation in o/w emulsions. Eur J Pharm Biopharm. 1996;42(2):104-109.

27. Müller RH. Zetapotential und Partikelladung in der Laborpraxis. Stuttgart, Germany: Wissenschaftliche Verlagsgesellschaft; 1996.

28. Bhalekar MR, Pokharkar V, Madgulkar A, Patil N, Patil N. Preparation and evaluation of miconazole nitrate-loaded solid lipid nanoparticles for topical delivery. AAPS Pharm Sci Tech. 2009;10(1): 289-296. 
29. Hou D, Xie C, Huang K, Zhu C. The production and characteristics of solid lipid nanoparticles (SLNs). Biomaterials. 2003;24(10):1781-1785.

30. Elsie O, Tiwari SB, Udupa N, et al. Niosome entrapped $\beta$-cyclodextrin methotrexate complex as a drug delivery system. Indian J Pharmacol. 1999;31(4):279-284.

31. Li H, Zhao X, Ma Y, Zhai G, Li L, Lou H. Enhancement of gastrointestinal absorption of quercetin by solid lipid nanoparticles. $J$ Control Release. 2009;133(3):238-244.

32. Castelli F, Puglia C, Sarpietro MG, Rizza L, Bonina F. Characterization of indomethacin-loaded lipid nanoparticles by differential scanning calorimetry. Int J Pharm. 2005;304(1-2):231-238.

33. Hu FQ, Jiang SP, Du YZ, Yuan H, Ye YQ, Zeng S. Preparation and characteristics of monostearin nanostructured lipid carriers. Int J Pharm. 2006;314(1):83-89.

34. Hou DZ, Xie CS, Huang KJ, Zhu C. The production and characteristics of solid lipid nanoparticles (SLNs). Biomaterials. 2003;24(10): 1781-1785.

35. Mogul MG, Akin H, Hasirci N, et al. Controlled release of biologically active agents for purposes of agricultural crop management. Resources, Conservation and Recycling. 1996;16(1-4):289-320.
36. Wissing SA, Mäder K, Müller RH. Prolonged efficacy of the insect repellent lemon oil by incorporation into solid lipid nanoparticles (SLN). Third World Meeting APGI/APV; Mainz, Germany: APV; 2000:439-440.

37. Wissing SA, Mäder K, Müller RH. Solid lipid nanoparticles (SLN) as a novel carrier system offering prolonged release of the perfume Allure (Chanel). Proceedings of the International Symposium on Controlled Release of Bioactive Materials; Paris, France; 2000:311-312.

38. Gao Z, Zhang D, Guo C. Paclitaxel efficacy is increased by parthenolide via nuclear factor-kappaB pathways in in vitro and in vivo human non-small cell lung cancer models. Curr Cancer Drug Targets. 2010; 10(7):705-715.

39. Feng L, Jia XB, Shi F, Chen Y. Identification of two polysaccharides from Prunella vulgaris $L$. and evaluation on their anti-lung adenocarcinoma activity. Molecules. 2010;15(8):5093-5103.

40. Zhang P, Chen L, Zhang Z, Lin L, Li Y. Pharmacokinetics in rats and efficacy in murine ovarian cancer model for solid lipid nanoparticles loading docetaxel. J Nanosci Nanotechnol. 2010;10(11):7541-7544.
International Journal of Nanomedicine

\section{Publish your work in this journal}

The International Journal of Nanomedicine is an international, peerreviewed journal focusing on the application of nanotechnology in diagnostics, therapeutics, and drug delivery systems throughout the biomedical field. This journal is indexed on PubMed Central, MedLine, CAS, SciSearch ${ }^{\circledR}$, Current Contents ${ }^{\circledR} /$ Clinical Medicine,

\section{Dovepress}

Journal Citation Reports/Science Edition, EMBase, Scopus and the Elsevier Bibliographic databases. The manuscript management system is completely online and includes a very quick and fair peer-review system, which is all easy to use. Visit http://www.dovepress.com/ testimonials.php to read real quotes from published authors. 